\title{
ON THE HANKEL DETERMINANTS OF CLOSE-TO-CONVEX UNIVALENT FUNCTIONS
}

\author{
K. INAYAT NOOR \\ Department of Mathematics \\ Kerman University \\ Kerman, Iran
}

(Received July 9, 1979 and in revised form August 29, 1979)

ABSTRACT. The rate of growth of Hankel determinant for close-to-convex functions is determined. The results in this paper are best possible.

KEY WORDS AND PHRASES. Starlike and close-to-convex Functions, Hankel Determinant 1980 MATHEMATICS SUBJECT CLASSIFICATION CODES. $30 A 32$.

1. INTRODUCTION.

Let $\mathrm{K}$ and $\mathrm{S}^{*}$ be the classes of close-to-convex and starlike functions in $\gamma=\{z:|z|<1\}$. Let $f$ be analytic in $\gamma$ and $f(z)=z+\sum_{n=2}^{\infty} a_{n} z^{n}$. The qth Hankel determinant of $\mathrm{f}$ is defined for $\mathrm{q} \geq 1, \mathrm{n} \geq 1$ by

$$
H_{q}(n)=\left|\begin{array}{cccc}
a_{n} & a_{n+1} & \ldots & a_{n+q-1} \\
a_{n+1} & \ldots & \ldots & \ldots \\
\vdots & & & \\
a_{n+q-1} & \ldots & \ldots & a_{n+2 q-2}
\end{array}\right|
$$


For $f \in S^{*}$, Pommerenke [2] has solved the Hankel determinant problem completely. Following essentially the same method, we extend his results in this paper to the class $\mathrm{K}$.

2. MAIN RESULTS.

THEOREM 1. Let $f \in K$ and $f(z)=z+\sum_{n=2}^{\infty} a_{n} z^{n}$. Then, for $m=0,1, \ldots$, there are numbers $\gamma_{m}$ and $c_{m \mu}(\mu=0, \ldots, m)$ that satisfy $\left|c_{m o}\right|=\left|c_{\operatorname{mm}}\right|=1$ and

$$
\sum_{k=0}^{\infty} \gamma_{k} \leq 3,0 \leq \gamma_{m} \leq \frac{2}{m+1}
$$

such that

$$
\sum_{\mu=0}^{m} c_{m \mu} a_{n+\mu}=0(1) n^{-1+\gamma_{m}} \quad(n \rightarrow \infty) .
$$

The bounds (2.1) are best possible.

PROOF. Since $f \in K$, there exists $g \in S^{*}$ such that, for $z \in \gamma$

$$
z f^{\prime}(z)=g(z) h(z), \operatorname{Reh}(z)>0
$$

Now $g$ can be represented as in $[1], g(z)=z \exp \left[\int_{0}^{2 \pi} \log \frac{1}{1-z e^{-1 t}} d \mu(t)\right]$, where $\mu(t)$ is an increasing function and $\mu(2 \pi)-\mu(0)=2$. Let $\alpha_{1} \geq \alpha_{2} \geq \ldots$ be the jumps of $\mu(t)$, and $t=\theta_{1}, \theta_{2}, \ldots$ be the values at which these jumps occur. We may assume that $\theta_{1}=0$. Then $\alpha_{1}+a_{2} \neq \ldots \leq 2$ and $\alpha_{1}+\alpha_{2}+\ldots+\alpha_{q}=2$ for some $q$ if and only if $g$ is of the form

$$
g(z)=z \prod_{j=1}^{q}\left(1-e^{-1 \theta} j z\right)^{\frac{-2}{q}}
$$

We define $\phi_{m}$ by

and

$$
\phi_{m}(z)=\prod_{\mu=1}^{m}\left(1-e^{i \theta_{\mu}} z\right)
$$

$$
\beta_{m}=a_{m+1}(m=0,1, \ldots)
$$

We consider the three cases i.e. 
(i) $0 \leq \alpha_{1} \leq 1$, (ii) $1<\alpha_{1}<\frac{3}{2}$, (iii) $\frac{3}{2} \leq \alpha_{1} \leq 2$

as in [2] and the first part, that is the bounds (2.1), follows similarly. For the rest, we need the following which is well-known [2].

LEMMA. Let $\theta_{1}<\theta_{2}<\ldots<\theta_{q}<\theta_{1}+2 \pi$, let $\lambda_{1}, \ldots, \lambda_{q}$ be real, and $\lambda>0, \lambda \geq \lambda_{j}(j=1, \ldots, q)$. If

$$
\begin{aligned}
& \psi(z)=\prod_{j=1}^{q}\left(1-e^{-i \theta_{j}} j_{j}-\lambda=\sum_{n=1}^{\infty} b_{n} z^{n}\right. \\
& \text { then } b_{n}=0(1) n^{\lambda-1} \quad \text { as } n \rightarrow \infty
\end{aligned}
$$

We write

$$
\phi_{m}(z)=\sum_{\mu=0}^{m} c_{m \mu} z^{m-\mu},
$$

and

$$
\phi_{m}(z) z f^{\prime}(z)=\sum_{n=1}^{m} b_{m n} z^{n+m}+\sum_{n=1}^{\infty}(n+m) a_{m n} z^{n+m}
$$

where

$$
\begin{aligned}
& b_{m}=\sum_{\nu=0}^{n}(n+v) c_{m-\nu} a_{n-v}, \\
& a_{m n}=\sum_{\mu=0} c_{m \mu} a_{n+\mu},\left|c_{m o}\right|=\left|c_{m m}\right|=1 .
\end{aligned}
$$

There are two cases.

(a) Let $g$ in (2.2) have the form (3); that is, $\alpha_{1}+\alpha_{2}+\ldots+\alpha_{q}=2$.

With $\gamma_{m}=\beta_{m}$, it follows that $\gamma_{m} \leq \frac{2}{m+1}, \gamma_{0}+\gamma_{1}+\ldots \leq 3$ and $\lambda_{m}=\frac{2}{m+1}$ implies $m=q-1, \alpha_{1}=\ldots=\alpha_{q}=\frac{2}{q}$.

Now from (2.2), (2.5) and the Cauchy Integral formula, we have, with

$$
\begin{aligned}
B_{m}(r)=\frac{1}{r^{m+n}} \sum_{k=1}^{m}\left|b_{m k}\right| r^{k+m}, \\
\quad(n+m)\left|a_{m n}\right| \leq \frac{1}{2 \pi r} \int_{0}^{2 \pi+m}\left|\phi_{m}(z) g(z) h(z)\right| d \theta+B_{m}(r) .
\end{aligned}
$$


Applying the Schwarz inequality, we have

$$
(n+m)\left|a_{m}\right| \leq \frac{1}{2 \pi r^{n+m}}\left(\int_{0}^{2 \pi}\left|\phi_{m}(z) g(z)\right|^{2} d \theta\right)^{\frac{1}{2}}\left(\int_{0}^{2 \pi}|h(z)|^{2} d \theta\right)^{\frac{1}{2}}+B_{m}(r) .
$$

When we write $\left[\phi_{m}(z) g(z)\right]^{2}$ in the form (2.4), the exponents $-\lambda_{j}$ satisfy $\lambda_{j} \leq 2 \gamma_{m}(j=1, \ldots q: m>0)$. Hence, using the Lemma, we have

$$
\int_{0}^{2 \pi}\left|\phi_{m}(z) g(z)\right|^{2} d \theta \leq \operatorname{An}^{2 \gamma_{m}-1}, \quad(n \rightarrow \infty) \text {. }
$$

Also

$$
\frac{1}{2 \pi} \int_{0}^{2 \pi}|h(z)|^{2} d \theta=\sum_{n=0}^{\infty}\left|d_{n}\right|^{2} r^{2 n}\left(d_{0}=1\right), \operatorname{Reh}(z)>0
$$

But $\left|d_{n}\right| \leq 2, n \geq 1$, and so

$$
\frac{1}{2 \pi} \int_{0}^{2 \pi}|h(z)|^{2} d \theta \leq 1+4 \sum_{n=1}^{\infty} r^{2} n=\frac{1+3 r^{2}}{1-r^{2}} \leq A n, n \geq 1
$$

From (2.7) and (2.8), we have

$$
(n+m)\left|a_{m n}\right| \leq A n^{\gamma_{m}} \quad(n \rightarrow \infty)
$$

i.e. $a_{m}=0(1) n^{\gamma_{m}^{-1}} \quad(n \rightarrow \infty)$.

This proves the theorem in this case.

(b) Let $g$ in (2.2) be not of the form (2.3). Then using arguments like those in $[2]$, it follows that, for $z=r e^{i \theta}$

$$
\int_{0}^{2 \pi}\left|\phi_{m}(z) g(z) h(z)\right| d \theta=0(1)(1-r)^{-\gamma_{m}} .
$$

Hence from (2.6), we have

$$
a_{m n}=0(1) n^{\gamma_{m}^{-1}}(n \rightarrow \infty),
$$

where $a_{m}$ is defined by (5). 
The function $f_{0}: f_{0}(z)=z\left(1-z^{q}\right)^{-2 / q}=\sum_{\nu=0}^{\infty}\left(_{\nu}^{2 / q+\nu-1}\right) z^{\nu q+1}$, shows that the bounds (1) are best possible. We also note that except in the case where $\mathrm{m}=(\mathrm{q}-1)$ and $\mathrm{g}$ in (2.2) is not of the form (2.3), one can choose $0 \leq \gamma_{\mathrm{m}}>\frac{2}{\mathrm{~m}+1}$ from theorem (1) and Pommerenke's method [2], we can now easily prove the following

THEOREM 2. Let $f \in K$ and $f(z)=z+\sum_{n=2}^{\infty} a_{n} z^{n}$. Then for $\mathrm{q} \geq 1, \mathrm{n} \geq 1$,

$$
H_{q}(n)=0(1) n^{2-q} \quad(n \rightarrow \infty)
$$

This estimate is best possible. In particular, if $g$ in (2.2) is not of the form (2.3), there exists a $\delta=\delta(q, g)>0$

such that $\mathrm{H}_{\mathrm{q}}(\mathrm{n})=0(1) \mathrm{n}^{2-\mathrm{q}-\delta} \quad(\mathrm{n} \rightarrow \infty)$.

\section{REFERENCES}

[1] Pommerenke, Ch. On Starlike and Convex Functions, J. London Math. Soc. 37 (1962) 209-224.

[2] Pommerenke, Ch. On the Coefficients and Hankel Determinants of Univalent Functions, J. London Math. Soc. 41 (1966) 111-122. 


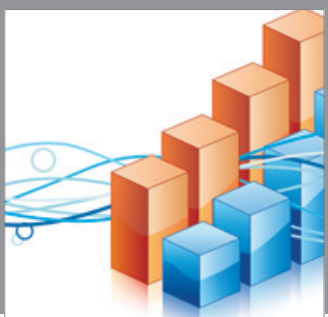

Advances in

Operations Research

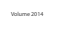

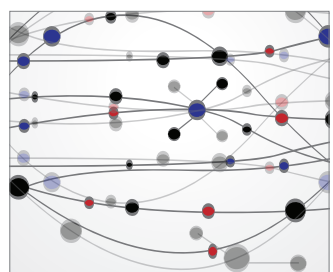

\section{The Scientific} World Journal
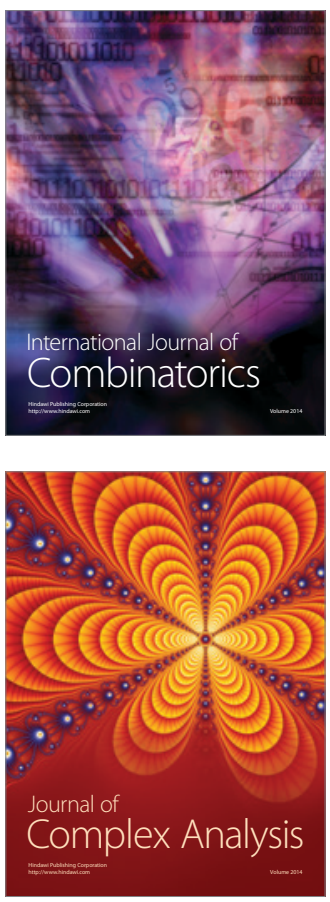

International Journal of

Mathematics and

Mathematical

Sciences
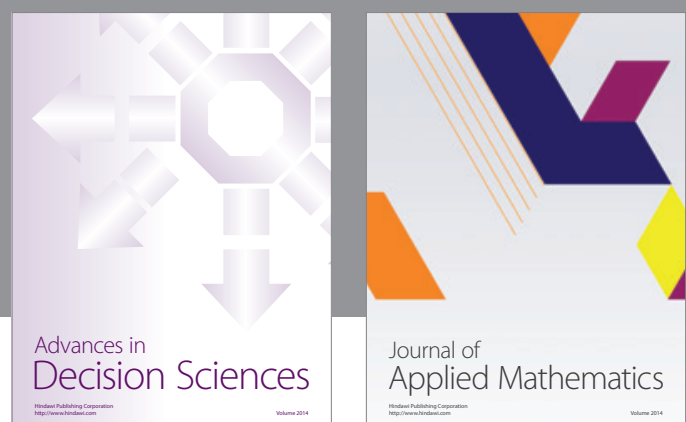

Journal of

Applied Mathematics
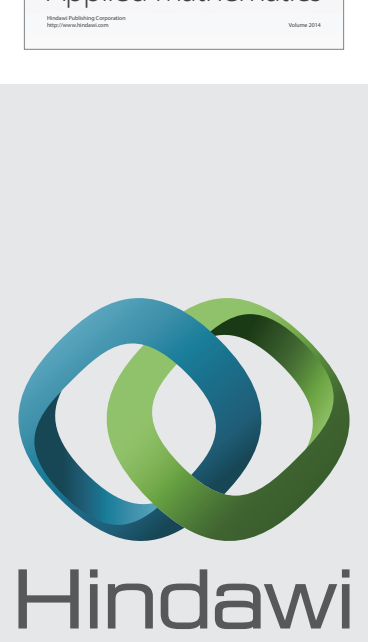

Submit your manuscripts at http://www.hindawi.com
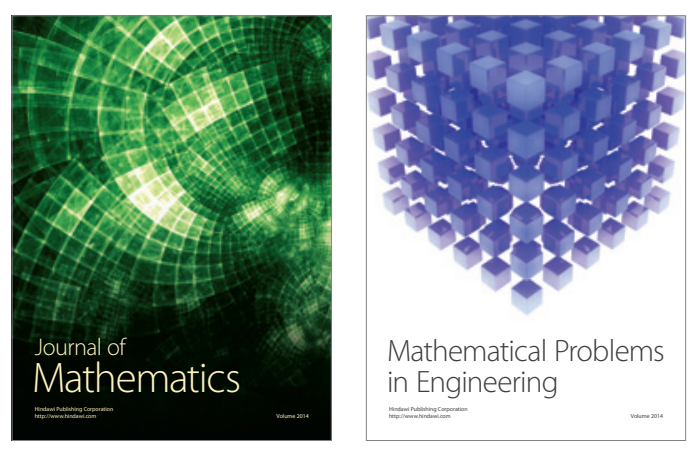

Mathematical Problems in Engineering
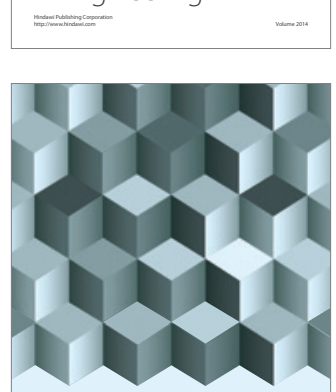

Journal of

Function Spaces
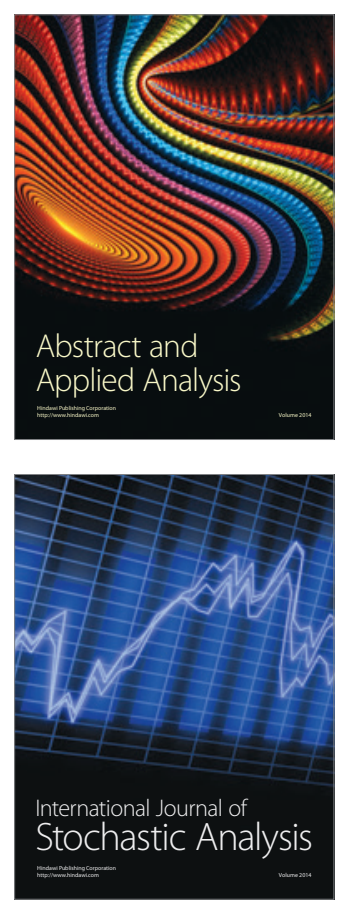

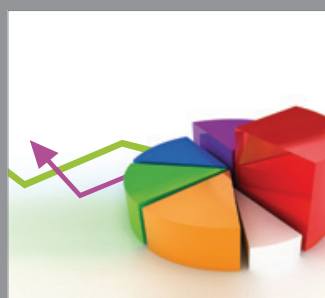

ournal of

Probability and Statistics

Promensencen
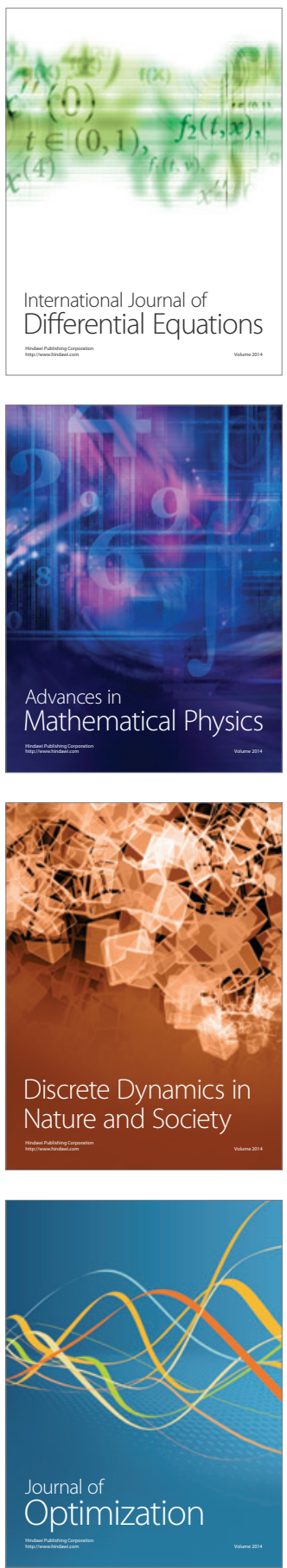